\title{
oints
}

$\sqrt{2}$

\section{The biomaterialist's task: scaffold biomaterials and fabrication technologies}

\author{
FRANCESCA GERVASO ${ }^{1}$, ALESSANDRO SANNINO' ${ }^{1}$, GIUSEPPE M. PERETTI ${ }^{2,3}$ \\ ${ }^{1}$ Department of Engineering for Innovation, University of Salento, Lecce, Italy \\ ${ }^{2}$ IRCCS Galeazzi Orthopaedics Institute, Milan, Italy \\ ${ }^{3}$ Department of Biomedical Sciences for Health, University of Milan, Italy
}

\begin{abstract}
This paper focuses on tissue engineering (TE) from the biomaterialist's point of view. With the aim of answering some simple but key questions about TE, the related literature is here reviewed. In order to obtain an engineered tissue the following steps are mandatory: (a) cell selection, (b) identification of the ideal three-dimensional scaffold for cell seeding and proliferation, (c) choice of the most suitable type of cell culture. Whereas the biotechnologist working in the TE field is responsible for optimizing the cell seeding and culture, the biomaterialist has the challenging task of optimizing the three-dimensional cell support, or scaffold. Therefore, in the present paper, scaffold properties, biomaterials and fabrication technologies are analyzed in depth and reviewed on the basis of the current literature. Finally, mention is also made of the most recently emerging and innovative technologies relating to scaffolds for TE applications.
\end{abstract}

Key Words: tissue engineering, natural polymer, bioceramics, scaffold production.

\section{Introduction}

It is exactly 20 years since tissue engineering (TE) was first presented as a promising new field that combined

\footnotetext{
Corresponding Author:

Giuseppe M. Peretti, MD

Department of Biomedical Sciences for Health,

University of Milan

Via Festa del Perdono 7, 20122 Milan, Italy

E-mail: gperetti@iol.it
}

the principles of biology and materials science in order to address one of the most important and still unresolved problems in human health, namely the loss or disease of organs and tissues (1).

Although TE has, ever since then, received more and more attention from the scientific community as a consequence of considerable investments by both government and private companies, it remains a new field whose rules and principles are still mostly undefined $(2,3)$.

The basic principles of TE are difficult to identify mainly because they are extremely dependent on the biological tissue needing to be replaced. In fact, research into the regeneration of some tissues such as, for example, human skin, has obtained significantly superior results compared with research into other tissues.

Pathologies of both the soft and hard tissues of the skeletal system, as a consequence of trauma or degeneration, are unfortunately very common and difficult to resolve. TE, in last two decades, has emerged as a promising approach to orthopedic repair (4-8).

That said, what is a TE approach? What are the basic principles underlying TE, and how it is possible to regenerate tissues using a TE approach?

In this review paper, we try to provide concise answers to these questions, focusing essentially on the two most important aspects for a biomaterialist: the biomaterials and the scaffold fabrication technologies.

As schematically reported in Figure 1, in order to induce tissue regeneration it is necessary (a) to select the type of cells to be used (primary or immortalized), (b) to choose a scaffold able to guarantee cell survival, proliferation and differentiation, (c) to establish which cell culture method to use (static or dynamic using a bioreactor). 


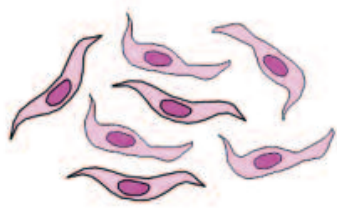

CELL SELECTION
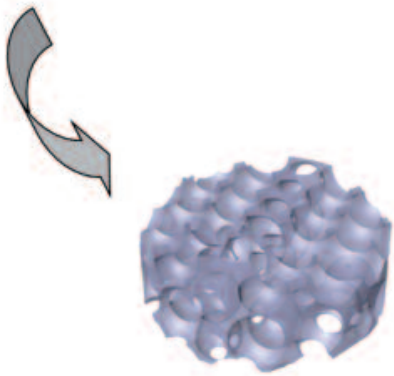

THREEDIMENSIONAL SCAFFOLD
TISSUE ENGINEERING

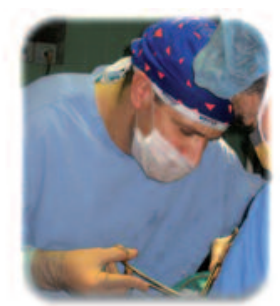

IN VIVO CULTURE, IMPLANT
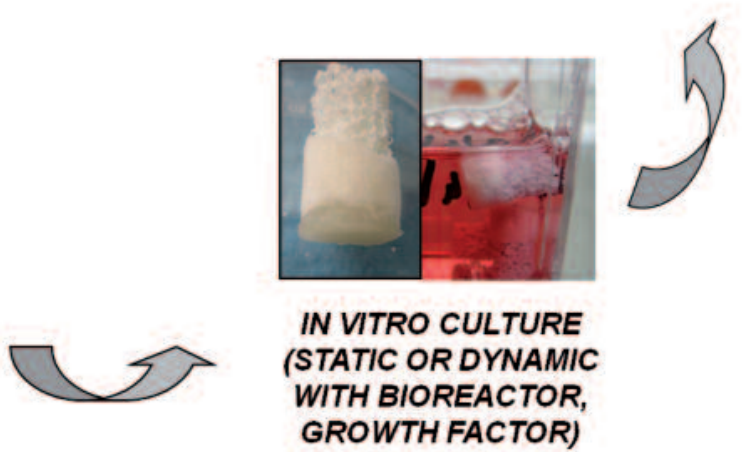

GROWTH FACTOR)

Fig. 1. Schematic representation of the tissue engineering approach for tissue regeneration.

While the main task of the biotechnologist is to optimize the cell seeding and culture, the biomaterialist's mission, instead, is to optimize the framework that has to support the cells, i.e. the scaffold. In order to optimize the scaffold, it is very important to define the properties that it must exhibit, and to be familiar with the materials that can be used and the technologies available for scaffold fabrication.

All these aspects are individually discussed in the following sections.

\section{Scaffold properties}

The scaffold is the three-dimensional structure onto and within which cells must adhere, proliferate, differentiate and produce extracellular matrix (ECM). In order to fulfill its advanced and sophisticated functions - it has to interact actively with the host's cells, transmitting signals useful for their sustenance - , it must be more than a mere mechanical support; for this reason, scaffolds have to exhibit very specific properties. Although scaffold properties are strictly related to the properties of the tissue that is to be regenerated, some of them, being fundamental, are common to all scaffolds. These common scaffold properties are: biocompatibility, biodegradability, mechanical strength and porosity (9).

\section{Biocompatibility}

A scaffold is biocompatible when it can be implanted in a human body without inducing any radical change in the intensity and duration of the optimal wound healing process $(10)$. The nature of the scaffold, i.e. biomaterial composition, surface charge and roughness, porosity and biodegradability, influences significantly the inflammatory response, as do the extent of the incision made for the implantation and the implant size and shape. A good biodegradable and biocompatible scaffold will allow new tissue replacement without inducing any fibrous capsular generation. A non-biocompatible scaffold is toxic to the host tissue, i.e. it can be rejected and cause death of the surrounding tissues. Biocompatibility tests, serving to establish the non-toxicity of a scaffold, are a mandatory preliminary step in a scaffold optimization process. 


\section{Biodegradability}

The scaffold should degrade in order to allow the new tissue to regenerate easily and restore the defect area. The degradation rate should match the tissue regeneration rate: the scaffold must not disappear too fast as it has to guarantee the important initial mechanical support; however, if scaffold degradation is too slow, its colonization by the new tissue could be compromised. The perfect scaffold remains as long as its scaffolding action is necessary for cell colonization, proliferation and differentiation, but it must have completely disappeared by the end of the regeneration process (9).

\section{Mechanical strength}

The mechanical properties of a scaffold should be as similar as possible to those of the host tissue needing to be regenerated. Since mechanical properties similar to those of the target tissue are not easily obtained, scaffolds should at least be able to withstand handling by the surgeon during implantation and the compressive and tensile loads occurring in the physiological environment. Although the above-described mechanical requirements are desirable for all TE applications, adequate mechanical strength is mandatory for orthopedic applications $(9,11)$. In order to determine accurately the mechanical properties of a scaffold, care must be taken to select the proper mechanical test. The composition and the function of the tissue needing to be restored will provide indications helping to determine the most suitable test to perform, i.e. compression or tensile, stress-relaxation, creep or stressstrain, static or dynamic, etc.

We wish to underline that making a scaffold with the appropriate mechanical properties is one of the crucial points in orthopedic TE and remains an open challenge.

\section{Porosity}

In order to allow (a) cell colonization and proliferation and (b) exchange of nourishment and waste products, the scaffold has to be highly porous and, moreover, feature a highly interconnected porosity. Pore size, too, plays an important role in cell survival and differentiation. The pore has to be big enough to allow cells to penetrate into the scaffold and to colonize the entire three-dimensional structure; at the same time, it must not be too big, as the scaffold is required to guarantee an adequate specific surface area necessary to provide a binding site for the cells. The ideal pore size will be strictly related to the site to be regenerated and to the cell type that is to be used. Therefore, it is not possible to indicate a single pore size suitable for all tissue engineered scaffolds $(9,11,12)$.

\section{Biomaterials}

One of the most important steps in the fabrication of a scaffold able to promote the regeneration of a tissue needing to be restored is the choice of material. Depending on the location, in the human body, of the tissue that needs to be replaced, some of the important properties illustrated above become essential and provide useful indications as to the material that should be used for the scaffold fabrication. To regenerate a large portion of bone tissue, for example, it is necessary to choose a material guaranteeing high mechanical performances even in the presence of high porosity. Many biomaterials have been used as scaffolds for cell culture and they can be divided into four main categories: natural polymers, synthetic polymers, bioceramics and composites $(9,13)$. Each class presents advantages and disadvantages that the biomaterialist must take into account in relation to the application field of interest.

In the next sections we list the main biomaterials in each class, and outline their main advantages, disadvantages and application fields.

\section{Natural polymers}

Natural polymers have been and continue to be the materials most used for scaffold fabrication because of their great similarity to the extracellular matrix structure and their biocompatibility and biodegradability (9). In fact, they can easily be modified, degraded and absorbed in vivo by enzymes naturally present in the human body (14).

Natural polymers are derived mainly from animal sources, but vegetable sources have also been investigated $(15,16)$. Among the large number of natural polymers of animal origin some have attracted particular interest in recent years, namely: collagen, gelatin, 
silk and chitosan. Instead, cellulose, alginate, agarose and cornstarch have been explored as potential scaffolds of vegetable origin.

Collagen is probably the most widely used natural polymer for TE applications. Many types of collagen are available, and they differ in terms of their animal source (i.e. bovine, equine, etc.) and zone of extraction (i.e. derma, tendon etc.). Collagen offers numerous advantages; first of all, it is present in most natural tissues, being the main component of connective tissue and the most abundant protein in the body, but it has the disadvantage of degrading very fast and at a quite uncontrolled rate, which reduces its mechanical strength (13). Crosslink treatments, thermal or chemical, can be performed in order to extend its durability in body fluids and increase its mechanical performance. Alternatively, composites made of collagen and a reinforcing phase, such as hydroxyapatite, have beenwidely proposed to improve the mechanical strength of the material (17-19).

When collagen is irreversibly hydrolyzed, gelatin is obtained. In very recent years, gelatin has been the focus of numerous investigations because it offers two important advantages: low cost and simple preparation (20). Because of its poor mechanical resistance, gelatin, especially in orthopedic applications, is usually used in combination with other materials, such as hydroxyapatite, bioactive glass, hyaluronic acid and chitosan $(16,20-22)$.

Silks are fibrous proteins produced by several species such as lepidoptera, mites, flies and spiders. In the last ten years, silks have been proposed as new scaffold biomaterials for TE applications due to their physical properties: they are stable, flexible and very resistant in tension and compression $(23,24)$. Because of the above-mentioned properties, silk-based scaffolds have been evaluated in depth for skeletal TE applications such as, for example, cartilage and bone regeneration $(25-27)$. The main disadvantage of silk is the very low speed of spider production.

Chitosan is obtained by the alkaline deacetylation of chitin, the world's second most abundant natural polysaccharide after cellulose. Chitin is extracted from exoskeletons of crustaceans and from cell walls of fungi or insects. Chitosan presents important properties that undoubtedly make it a biomolecule offering great potential for regenerative medicine. Among the several properties of chitosan we cite its biodegradability, high adsorption capacity, gel forming ability and the presence of reactive functional groups (28).

\section{Synthetic polymers}

Although natural polymers are currently the materials most extensively used in the fabrication of scaffolds, synthetic polymers have been and continue to be widely used since they have good mechanical strength, reproducible/controllable mechanical-chemical properties, and controllable biodegradation rates.

Synthetic polymers can be divided in two classes: biodegradable, such as poly(glycolic), poly(lactic)acid and copolymers, polycaprolactone, polydioxanone, and polyurethanes, and non-biodegradeable, such as polyvinyl alcohol, polyhydroxyethymethacrylate, and poly(N-isopropylacrylamide).

However, because the most common synthetic polymers have ahydrophobic nature, they show poor wetting properties that result in poor cell distribution and poor cell adhesion. Moreover, synthetic polymers can cause systemic or local reactions caused by acidic degradation products.

In order to improve the cell affinity of these materials, surface treatments have recently been proposed and are currently under examination by numerous investigators $(29,30)$.

\section{Bioceramics}

A ceramic material used in a biomedical application can be defined a bioceramic material. Bioceramics is the science governing the production of solid ceramic bodies, porous or dense, by the melting of raw inorganic material (9). The main application field of bioceramic materials is orthopedics because of the great affinity of the chemical composition of some ceramic materials with the mineral constituent of bones. The bioceramic materials can be grouped into three classes according to their behavior in the physiological environment: (a) bioresorbable, i.e. calcium and three-calcium phosphates, (b) bioactive or surface-active, i.e. hydroxyapatite, (c) non-resorbable or inert, i.e. alumi$n a$ and zirconia.

The main disadvantages of these materials are their low mechanical resistance and high brittleness; their 
main advantagesare their high biocompatibility and good osteconductivity.

\section{Composites}

One of the most interesting solutions currently under evaluation in order to overcome some of the disadvantages of the single-phase materials described above is the combination of different materials to obtain a composite. A composite material should increase the performance of the single-phase materials that compose it. Among the possible combinations, we cite: (a) natural or synthetic polymer-ceramic, and (b) polymer-polymer, both natural and synthetic. The main advantage of a composite is that it can provide tailored mechanical properties and degradation rates. The main disadvantage is that the scaffold fabrication techniques can be quite complicated.

\section{Scaffold fabrication technologies}

The spatial organization of tissue in the human body is prevalently three-dimensional. For this reason, a scaffold capable of restoring diseased or missing tissue inside the body has to be three-dimensional too. The aim of the most innovative technologies used for scaffold fabrication is therefore to obtain three-dimensional structures with a high and a highly interconnected porosity in order to resemble, as closely as possible, the native tissue (31).
Numerous fabrication methods have been reported in the literature over the past ten years. Of these, freeze drying, particulate leaching, sponge replication, rapid prototyping, gas foaming and microsphere sintering are the most used and consolidated.

\section{Freeze drying}

In freeze drying, the material of interest is dispersed in a liquid phase in order to obtain a solid phase homogenously dispersed in a liquid phase. The slurry is then frozen and subsequently oven dried under high vacuum conditions in order to allow the solid-gas phase transformation. The result will be a highly porous scaffold featuring high interconnection of the porosity.

In order to control the percentage and orientation of the porosity, pore size, architecture, and mechanical properties, compositional and process parameters can be varied (32). In this way, it is possible to obtain the desired anisotropic orientation of the porosity (Fig. 2).

\section{Particulate leaching}

Particulate leaching consists of dissolving a polymeric material in an organic solvent and adding particles, usually salts, to the solution. The solution is then poured into a mold and the solvent is allowed to evaporate. The polymer-particle composite material is then placed in a bath able to dissolve the particles; this results in a porous three-dimensional polymeric structure.

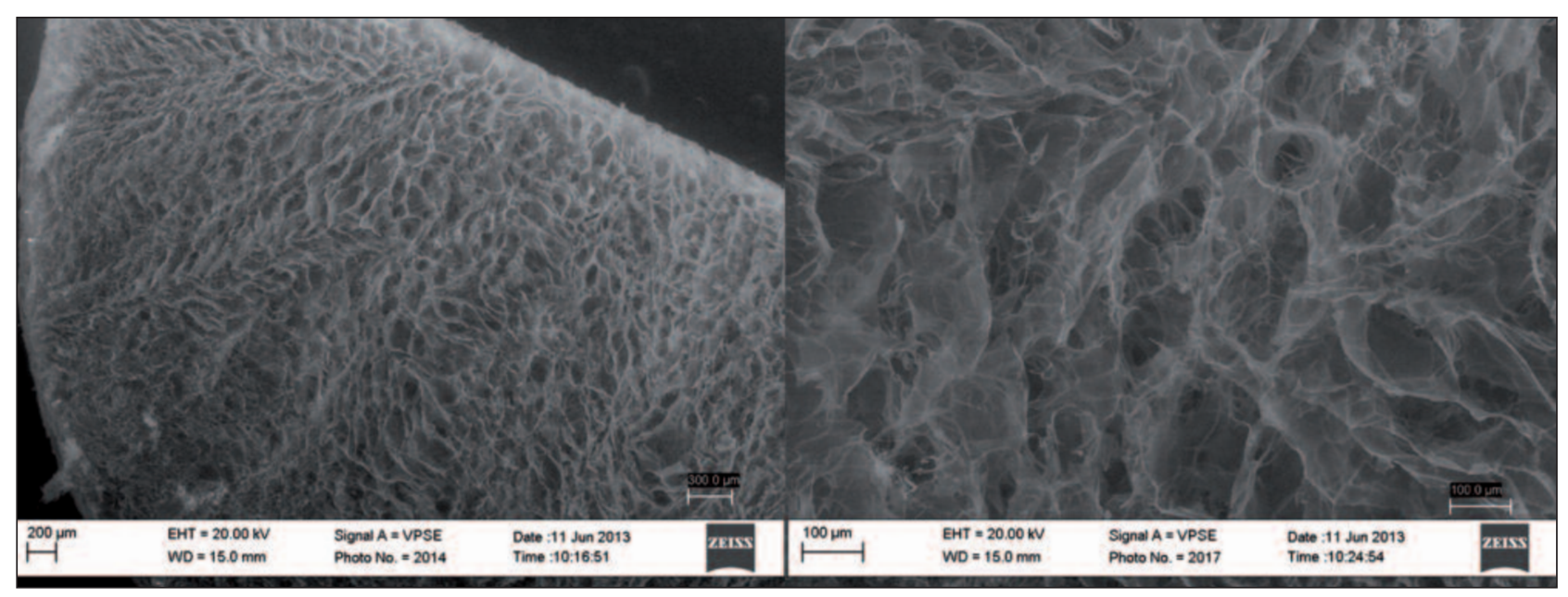

Fig. 2. SEM images of a chitosan scaffold fabricated using the freeze-drying technique at two different magnifications. 
This technique is widely used for the fabrication of highly porous scaffolds made of synthetic polymers (33).

\section{Sponge replication}

In order to obtain a highly porous three-dimensional scaffold in a ceramic material the sponge replication method can be used. This consists of impregnation of a polymeric sponge with a ceramic slurry (ceramic powder suspended in a liquid phase) and subsequent heat treatment at high temperature of the infiltrated sponge in order to allow burn-out of the sponge and, following a further increase of the temperature, sintering of the ceramic.

The result is a ceramic sponge that perfectly replicates the geometry of the original sponge. Selecting or forming a sponge having the geometry of interest, the replication method makes it possible to obtain a ceramic scaffold having that exact same geometry (34) (Fig. 3).

\section{Rapid prototyping}

Rapid prototyping is a technique that allows the quick fabrication of three-dimensional parts starting from CAD (computer-aided design) data. The fabrication of the object is usually performed using three-dimensional printing technologies such as fused deposition modeling (FDM). FDM has the potential to design and fabricate highly reproducible 3D scaffolds with fully interconnected pore networks, building physical models by depositing layers of a thermoplastic material one at a time (35).

\section{Gas foaming}

The gas foaming process consists of pressurizing a polymeric solution (in water or fluoroform) at highpressures with a gas-foaming agent, such as $\mathrm{CO}_{2}$ and nitrogen, until the polymers are saturated. As a consequence of the high pressurization, nucleation and growth of gas bubbles will occur, generating pores inside the polymeric structure. This technique has the advantage of being an organic solvent-free process, butthe drawback of generating structures with a basically unconnected porosity. For this reason, in the literature, the combination of gas foaming with salt leaching techniques has been proposed (36).

\section{Microsphere sintering}

Microsphere sintering is a very simple technique in which polymeric or ceramic microspheres falling within a narrow size range are thermally fused together to form a three-dimensional porous structure.This structure can itself be used as a scaffold, or as synthetic scaffold providing a negative template for, for example, bone regeneration (37) (Fig. 4).

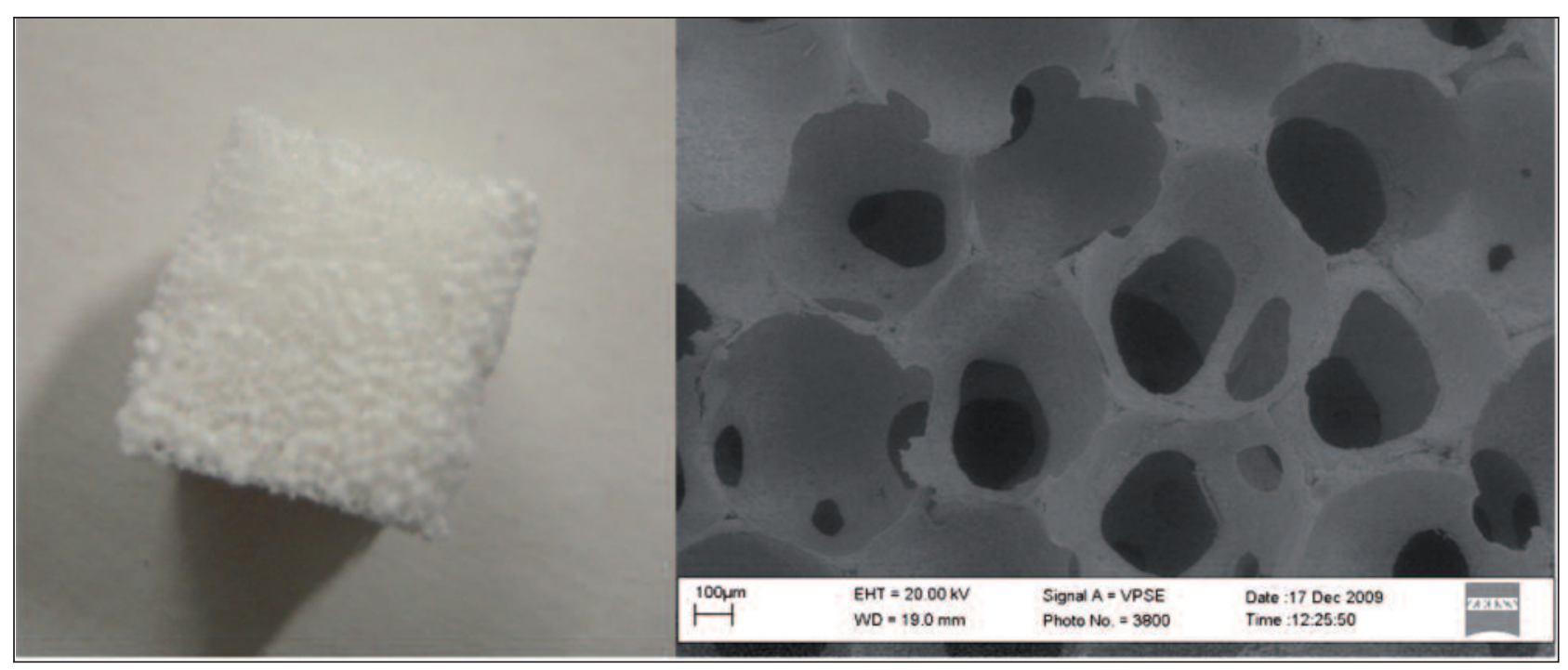

Fig. 3. An example of a hydroxyapatite scaffold obtained using the sponge replication method. A picture of the scaffold (left) and SEM image of the macrostructure of the ceramic sponge (right). 


\section{oincts}

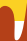

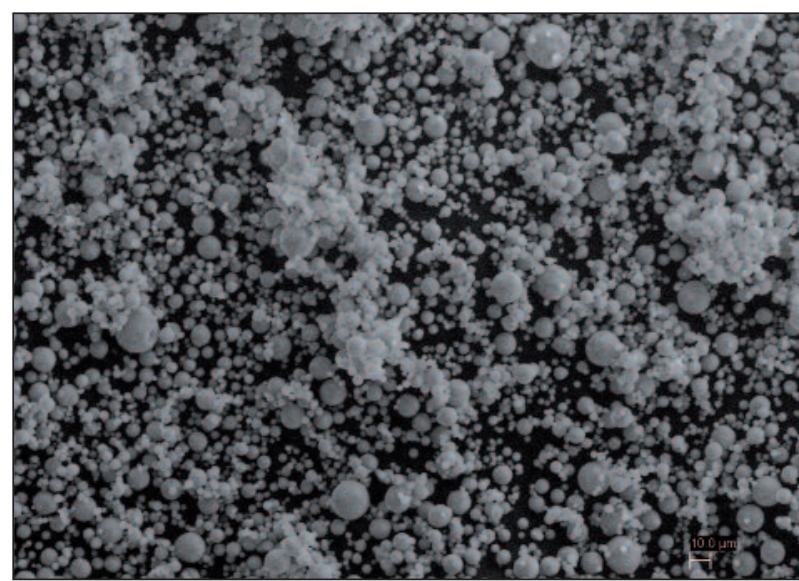

Fig. 4. Scanning electron micrographs showing hydroxyapatite microspheres produced by spraydrying.

\section{New frontiers}

Although the above illustrated techniques make it possible to obtain geometries of increasing complexity using innovative materials, the resulting scaffolds are still not able to actively modulate cell behavior or mimic the complex interaction between the cells of a specific tissue and the extracellular matrix of that specific tissue.

The capacity to develop active scaffolds that are able to 'dialogue' with cells is important in order to achieve significant advancement of the TE field. In order to obtain such smart scaffolds the currently used scaffold fabrication technologies must be integrated with the more innovative techniques of surface modification. In the last few years, the importance of the properties of the scaffold surface, together with the possibility of modifying it, has emerged as a key factor in TE advancement.

Numerous investigators are currently working on scaffold surface treatment via plasma or using nanotechnologies in order (a) to modify the surface to improve the affinity between cell and substrate (i.e. hydrophilization of hydrophobic material), (b) to cover the surface with a more affine material by deposition, (c) to add receptor sites to the surface in order to link drug/growth factor release systems (38).

In conclusion, we still do not have the perfect scaffold for each TE application. Moreover, the fabrication techniques so far developed are still not able to give us an active, smart scaffold capable of dialoguing with cells. However, the most recent surface treatment techniques based mostly on nanoscale technologies will surely offer new and very promising solutions representing a step forward in the TE field (39).

\section{References}

1. Langer R, Vacanti JP. Tissue engineering. Science 1993;260:920-926.

2. Williams D. Benefit and risk in tissue engineering. Materials Today2004,7: 24-29.

3. Yannas IV. Emerging rules for inducing organ regeneration. Biomaterials 2013;34: 321-330.

4. Tucker B, Khan W, Al-Rashid M, Al-Khateeb H. Tissue engineering for the meniscus: areview of the literature. Open Orthop J 2012;6:348-351.

5. Longo UG, Lamberti A, Petrillo S, Maffulli N, Denaro V. Scaffolds in tendon tissue engineering. Stem Cells Int 2012, 2012; 517165 .

6. Lu HH, Subramony SD, Boushell MK, Zhang AX. Tissue engineering strategies for the regeneration of orthopedic interfaces. Ann Biomed Eng 2010;38:2142-2154.

7. Nukavarapu SP, Dorcemus DL. Osteochondral tissue engineering: current strategies and challenges. Biotechnol Adv 2013;31:706-721.

8. Zhang X, Bogdanowicz D, Erisken C, Lee NM, Lu HH. Biomimetic scaffold design for functional and integrative tendon repair. J Shoulder Elbow Surg 2012;21:266-277.

9. Garg T, Singh O, Arora S, Murthy R. Scaffold: anovel carrier for cell and drug delivery. Crit Rev Ther Drug Carrier Syst 2012;29:1-63.

10. Mikos AG, McIntire LV, Anderson JM, Babensee JE. Host response to tissue engineered devices. Adv Drug Deliv Rev 1998;33:111-139.

11. O' Brien FJ. Biomaterials and scaffolds for tissue engineering. Materials Today 2011;14:88-95.

12. Billström GH, Blom AW, Larsson S, Beswick AD. Application of scaffolds for bone regeneration strategies: current trends and future directions. Injury 2013;44 Suppl $1: S 28-S 33$.

13. Zanetti AS, Sabliov C, Gimble JM, Hayes DJ. Human adipose-derived stem cells and threedimensional scaffold constructs: a review of the biomaterials and models currently used for bone regeneration. J Biomed Mater Res B Appl Biomater 2013;101:187-199.

14. Gaalen S. Tissue engineering of bone. In: van Blitterswijk (ed), Tissue Engineering. Elsevier Academic Press, San Diego 2008;559-610.

15. KoHF, Sfeir C,Kumta PN. Novel synthesis strategies for natural polymer and composite biomaterials as potential scaffolds for tissue engineering. Philos Trans A Math Phys Eng Sci 2010;368:1981-1997.

16. Khan MN, Islam JM, Khan MA. Fabrication and characterization of gelatin-based biocompatible porous composite scaffold for bone tissue engineering. J Biomed Mater Res A 2012;100:3020-3028.

17. Zhou J, Xu C, Wu G, Cao X, Zhang L, Zhai Z, Zheng Z, Chen X, Wang Y. In vitro generation of osteochondral differentiation of human marrow mesenchymal stem cells in novel 
collagen-hydroxyapatite layered scaffolds. Acta Biomater 2011;7:3999-4006.

18. Prosecká E, Rampichová M, Vojtová L, Tvrdík D, Melčáková S, Juhasová J, Plencner M, Jakubová R, Jančář J, Nečas A, Kochová P, Klepáček J, Tonar Z, Amler E. Optimized conditions for mesenchymal stem cells to differentiate into osteoblasts on a collagen/hydroxyapatite matrix. J Biomed Mater Res A 2011;99:307-315.

19. Sionkowska A, Kozłowska J. Properties and modification of porous 3-D collagen/hydroxyapatite composites. Int J Biol Macromol 2013;52:250-259.

20. Ko CL, Tien YC, Wang JC, Chen WC. Characterization of controlled highly porous hyaluronan/gelatin cross-linking sponges for tissue engineering. J Mech Behav Biomed Mater 2012;14:227-238.

21. Chiu CK, Ferreira J, Luo TJ, Geng H, Lin FC, Ko CC. Direct scaffolding of biomimetic hydroxyapatite-gelatin nanocomposites using aminosilane cross-linker for bone regeneration. J Mater Sci Mater Med 2012;23:2115-2126.

22. Nadeem D, Kiamehr M, Yang X, Su B. Fabrication and in vitro evaluation of a sponge-like bioactive-glass/gelatin composite scaffold for bone tissue engineering. Mater Sci Eng C Mater Biol Appl 2013;33:2669-2678.

23. Altman GH, Diaz F, Jakuba C, Calabro T, Horan RL, Chen J, Lu H, Richmond J, Kaplan DL. Silk-based biomaterials. Biomaterials 2003;24:401-416.

24. MacIntosh AC, Kearns VR, Crawford A, Hatton PV. Skeletal tissue engineering using silk biomaterials. J Tissue Eng Regen Med 2008;2:71-80.

25. Wang Y, Blasioli DJ, Kim HJ, Kim HS, Kaplan DL. Cartilage tissue engineering with silk scaffolds and human articular chondrocytes. Biomaterials 2006;27:4434-4442.

26. Bhardwaj N, Kundu SC. Chondrogenic differentiation of rat MSCs on porous scaffolds of silk fibroin/chitosan blends. Biomaterials 2012;33:2848-2857.

27. Mandal BB, Grinberg A, Gil ES, Panilaitis B, Kaplan DL. High-strength silk protein scaffolds for bone repair. Proc Natl Acad Sci USA 2012;109:7699-7704.

28. Shi C, Zhu Y, Ran X, Wang M, Su Y, Cheng T. Therapeutic potential of chitosan and its derivatives in regenerative medicine. J Surg Res 2006;133:185-192.
29. Poncin-Epaillard F, Shavdina O, Debarnot D. Elaboration and surface modification of structured poly(L-lactic acid) thin film on various substrates. Mater Sci Eng C Mater Biol Appl 2013;33:2526-2533.

30. Ajiro H, Takahashi Y, Akashi M, Fujiwara T. Polylactide block copolymers using trimethylene carbonate with methoxyethoxy side groups for dual modification of hydrophilicity and biodegradability. MacromolBiosci 2012;12:1315-1320.

31. Lu T, Li Y, Chen T. Techniques for fabrication and construction of three-dimensional scaffolds for tissue engineering. Int J Nanomedicine 2013;8:337-350.

32. Ma PX, Zhang R.Microtubular architecture of biodegradable polymer scaffolds. J Biomed Mater Res 2001; 56:469-477.

33. Oh SH, Kang SG, Kim ES, Cho SH, Lee JH. Fabrication and characterization of hydrophilic poly(lactic-co-glycolic acid)/ poly(vinyl alcohol) blend cell scaffolds by melt-molding particulate-leaching method. Biomaterials 2003;24:4011-4021.

34. Gervaso F, Scalera F, Sanosh KP, Sannino A, Licciulli A. High-performance hydroxyapatite scaffolds for bone tissue engineering applications. Int J Appl Ceram Tec 2012;9:507516.

35. Hutmacher DW, Schantz T, Zein I, Ng KW, Teoh SH, Tan KC. Mechanical properties and cell cultural response of polycaprolactone scaffolds designed and fabricated via fused deposition modeling. JBiomedMater Res 2001;55:203-216.

36. Nam YS, Yoon JJ, Park TG. A novel fabrication method of macroporous biodegradable polymer scaffolds using gas foaming salt as a porogen additive. J Biomed Mater Res 2000; 53:1-7.

37. Borden M, El-Amin SF, Attawia M, Laurencin CT. Structural and human cellular assessment of a novel microsphere-based tissue engineered scaffold for bone repair. Biomaterials 2003;24:597-609.

38. Lee SJ, Atala A. Scaffold technologies for controlling cell behavior in tissue engineering. Biomed Mater 2013;8: 010201.

39. Saiz E, Zimmermann EA, Lee JS, Wegst UG, Tomsia AP. Perspectives on the role of nanotechnology in bone tissue engineering. Dent Mater 2013;29:103-115. 